\title{
The Impact of Fractal Geometry on Permittivity and Related Quantities
}

\author{
Dubravko Risovića,*, Blaženka Gašparovićb, Božena Ćosovićb \\ ${ }^{a}$ Molecular Physics Laboratory, Ruđer Bošković Institute, POB 180, HR-10002 Zagreb, \\ Croatia and ${ }^{b}$ Center for Marine and Environmental Research, Ruđer Bošković Institute, \\ POB 180, HR-10002 Zagreb, Croatia.
}

A new physical model of relative permittivity and derived quantities of fractal structures, contrary to the current concept, predicts their dependence on the fractal dimension $(D)$ and on the extent $(R)$ of the considered structure. The scaling of considered quantities predicted by our theory is $\sim R^{\mathrm{D}-\mathrm{d}}$, where $d$ is dimension of Euclidean space. Hence, fractality, a feature often found in physical, chemical and biological systems, influences also basic properties of such systems that, so far, were believed to be material and not structure related. The theory has been experimentally verified by electrochemical measurements of capacitance of adsorbed layers of nonionic surfactant Triton-X-100 and linoleic acid that have fractal structure. The experimental results, substantiating theoretical predictions, are presented and the influence of relevant parameters is discussed.

${ }^{*}$ Corresponding author. Tel.: +385-1-456-11-22; Fax: +385-1-468-01-12.

E-mail:drisovic@rudjer.irb.hr 


\section{Introduction}

The dielectric features of material, relative permittivity, susceptibility, polarizibility and related properties such as capacitance play a significant role in various chemical, physical and biological systems, and on scales ranging from microscopic to macroscopic. Among other things capacitance is link to the knowledge of the fluctuations of several physical quantities, e.g. voltage and electromagnetic field fluctuations, ${ }^{1-6}$ dipole moment, ${ }^{5-7} \mathrm{pH}$ and charge, ${ }^{8}$ and also to polarizibility and dielectric dispersion of colloidal and polyelectrolite systems ${ }^{5-7}$. Hence, it is crucial for understanding of biological systems that include ionic channels and cell membranes, ${ }^{9-11}$ but also other systems such as adsorbed and spread films ${ }^{12-14}$. On the other hand it has been shown that many of these systems are fractal structures or exhibit fractal behavior, ${ }^{15}$ and can be described with effective fractal dimension $D$. Therefore it was worthwhile to examine the possible influence of fractal geometry on fundamental and derived electric properties. In that context we examine the relative permittivity and specific capacitance of fractal structures and their dependence on geometrical features. The notion of these quantities in classical (non-fractal) system implies that they are constant and characteristics of the material of the considered system be it physical, chemical or biological.

Starting from considerations of fundamental material properties we have developed a general theoretical model that predicts dependence of relative permittivity and consequently $\mathrm{C}_{\mathrm{s}}$ of fractal structures (e.g. adsorbed layer, molecular or particle aggregates, porous material etc.) on structure size and fractal dimension. These theoretical predictions have been experimentally verified by electrochemical measurements of capacitance of adsorbed layers possessing fractal structure, in 
conjunction with recently introduced method for determination of fractal dimension of such layers ${ }^{16}$. This specific testbed was chosen because structural and dynamic properties of adsorbed molecular films are of both fundamental and applied interest in diverse areas. These systems were studied with various techniques, as optical, ${ }^{17-20}$ electrochemical, ${ }^{21,} 22$ atomic force microscopy, ${ }^{23,} 24$ etc. Although, most of the adsorption studies deal with the fractality of the surface ${ }^{25-28}$ there is also evidence of a fractality of the adsorbed layer itself. ${ }^{16,29,30}$

The approach used in study of fractal structures is based on the possibility to describe quantitatively complex objects that are statistically scale-invariant, physical realizations of mathematical fractals that appear the same on all length scales. This property manifests itself as a power-law-scaling ratio that characterizes one or more features of an object or a process carried out near or at the object:

$$
\text { Feature } \sim \text { scale }^{\Delta}
$$

Here "feature" should be considered in the broadest sense (e.g. surface area, scattered light intensity, the rate of reaction etc.) and the "scale" could be particle size, pore diameter, scattering vector, cross-sectional area of an adsorbate or layer thickness. The exponent $\Delta$ is a parameter, which indicates how sensitive the considered feature is to changes in the applied scale. This non-integer exponent $\Delta$ has the meaning of dimension, which Mandelbrot ${ }^{15}$ termed "fractal". Extension of this concept, coming from the recognition that effective geometries of various structures and processes can be described in terms of fractal geometry, results in an effective fractal dimension $D_{\text {feature }}$ or simply $D$.

The geometrical properties of material may play significant role in a various processes and reflect themselves on macroscopic level properties. On the other hand, various processes especially self-organizational processes may result in a fractal 
structures with unusual features on micro- and macroscopic level. Hence, we had assumed that geometrical features, such as fractality, should reflect itself also in basic material properties, such as dielectric properties.

\section{Theory}

In order to develop relation between basic dielectric properties and fractal geometry we proceed by examining the basic molecular properties of such structures.

In a dielectric medium the displacement vector, $\vec{D}$, is given by

$$
\vec{D}=\varepsilon_{0} \vec{E}+\vec{P}
$$

where $\vec{E}$ is electric field, $\vec{P}$ is polarization (electric dipole moment per unit volume), and $\varepsilon_{0}$ is permittivity of free space $\left(\varepsilon_{0}=8.85410^{-12} \mathrm{~F} / \mathrm{m}\right)$.

On a macroscopic level the polarization is defined by:

$$
\vec{P}=\chi \varepsilon_{0} \vec{E}_{e}
$$

where $E_{e}$ is the external field, $\chi$ is the electric susceptibility, a dimensionless scalar.

In a dense media with closely packed molecules the polarization of neighboring molecules give rise to an internal microscopic field, $E_{m}$, at any given molecule in addition to the average macroscopic field. Hence, microscopically:

$$
\vec{P}=N \alpha \vec{E}_{m}
$$

where $\alpha$ is polarizibility and $N$ is molecular density (number of molecules per unit volume). 
Connection with the macroscopic field is given by: ${ }^{31}$

$$
\vec{P}=\frac{N \alpha / \varepsilon_{0}}{1-\frac{N \alpha / \varepsilon_{0}}{3}} \varepsilon_{0} \vec{E}_{e}
$$

wherefrom we get:

$$
P=\frac{N \alpha E_{e}}{1-\frac{N \alpha}{3 \varepsilon_{0}}}
$$

The relative permittivity (dielectric constant), $\kappa$, is given by:

$$
\kappa=1+\frac{P}{\varepsilon_{0} E_{e}}
$$

Introduction of Eq. 5a into Eq. 6 gives:

$$
\kappa=1+\frac{N \alpha}{\varepsilon_{0}}\left(1-\frac{N \alpha}{3 \varepsilon_{0}}\right)^{-1}
$$

If we use the expansion:

$$
\left(1-\frac{N \alpha}{3 \varepsilon_{0}}\right)^{-1}=1+\frac{N \alpha}{3 \varepsilon_{0}}+\left(\frac{N \alpha}{3 \varepsilon_{0}}\right)^{2}+\ldots
$$

and retain only linear term one gets:

$$
\kappa \approx 1+\frac{N \alpha}{\varepsilon_{0}}
$$

This relation is generally valid (in a week polarisability approximation) and does not imply any structural effects.

Now, if we consider a fractal structure made from molecules, particles, etc. (either in three or two dimensions) then, the number of particles, $n$, within any space of size $R$ is $n(R) \sim R^{D}$, where $D$ is the fractal dimension. The average density of particles (number of particles per unit surface or volume) is given by:

$$
N \propto \mathrm{n}(R) / R^{\mathrm{d}}
$$


where $d$ is the dimension of a space containing considered fractal structure.

Hence, for a fractal structure of size, $R$, the density, $N$, varies as:

$$
N \propto R^{\mathrm{D}-\mathrm{d}}
$$

For $D=d$ we obtain the familiar result that the average density is independent of considered size.

Now, using Eq. 8 and 10, the dielectric constant of the fractal structure is given by:

$$
\kappa-1 \propto R^{D-d}
$$

where, the proportionality constant includes also the term $\alpha / \varepsilon_{0}$. This relation clearly shows that the relative permittivity is not only a characteristic of the material but for fractal structures also depends on extent and $D$ of the considered structure. Consequently, for a fractal structure all physical quantities related to the relative permittivity, such as polarization and specific capacity, shall exhibit similar scaling.

To verify this theoretical prediction we now apply this theory to an electrochemical system. We shall consider the capacitance of adsorbed layers that exhibit fractal properties. Such systems are usually modeled by parallel plate capacitor. Consider a capacitor that between its plates contains a material characterized by $\kappa$. Its capacitance, $C$, is enhanced in regard to the capacitor without the material (vacuum) whose capacitance is $C_{0}$ :

$$
C=C_{0} \kappa
$$

Similar relation holds for the specific capacitance (capacitance per unit area)

$$
C_{s}=C_{0 s} \kappa
$$

If we consider the $C_{s}$ of a fractal structure (e.g. fractal layer adsorbed on an electrode surface or a capacitance of a fractal membrane), using Eq. (11) and including the appropriate proportionality constant, one obtains: 


$$
C_{s}=C_{0 s}+K R^{D-d}
$$

where $K=k C_{0 s} \frac{\alpha}{\varepsilon_{0}}$, and $k$ denotes the proportionality constant in Eq. 10. Insight into the physical meaning of proportionality constant $K$ can be attained through dimensional analysis of Eq. 14. For $d=2$ it follows: $\left[C_{s}\right]=\left[C_{0 s}\right]+[K] L^{D-2}$. The dimension of a quantity is denoted by square bracket. Hence, the dimension of $[\mathrm{K}] \mathrm{L}^{\mathrm{D}-2}$ should be equal to the dimension of the capacitance, or: $M^{-1} L^{-2} t^{2} q^{2} L^{-2}=[K] L^{D-2}$, where $M, L, t, q$ are dimensions of mass, length, time and charge, respectively. Therefore $[K]=[C] L^{-D}$ with $[C] \equiv M^{-1} L^{-2} t^{2} q^{2}$ (dimension of capacitance). Hence, $K$ has a dimension of capacitance per unit fractal space ("area") of dimension $D$, and could be called "the specific fractal capacitance".

Hence, for $D=d$ the specific capacitance of the considered system shall be constant and independent on size. This result complies with the classical notion and definition of specific capacitance. However, for a fractal structure with $D \neq d$ the capacitance per unit surface area, $C_{s}$, is size dependent and scales as $R^{\mathrm{D}-\mathrm{d}}$.

Now we apply this theory to an electrode/electrolyte interface with adsorbed layer on the hanging mercury drop electrode (HMDE). The considered system is spherical, hence, in the exact treatment the relation for the capacitance of a spherical capacitor should be applied. However, due to different scales of involved dimensions (radii of mercury drop and thickness of the Helmholtz layers), the model of parallel plate capacitor represents a good approximation.

The differential capacitance, $C$, of the interface, in approximation of parallel plate capacitor, is given by:

$$
C=\frac{\varepsilon_{0} \kappa A}{l}
$$


where $A$ is area and $l$ is separation of the plates.

The specific capacitance is given by:

$$
C_{s}=\frac{C}{A}=\frac{\varepsilon_{0} \kappa}{l}
$$

Use of Eq. 16 instead of Eq. 13 leads to the modified Eq. 14 adequate for the considered electrochemical system:

$$
C_{s}=\frac{\varepsilon_{0}}{l}\left(1+\frac{\alpha}{\varepsilon_{0}} k R^{D-d}\right)
$$

wherefrom:

$$
C_{s}=\frac{\varepsilon_{0}}{l}+\frac{\alpha}{l} k R^{D-d}
$$

designating $\varepsilon_{0} / l=C_{0 s}$, and $(\alpha k / l)=\beta$, a specific equation for $C_{s}$ of the fractal interface, an equivalent to the general Eq. 14, is obtained:

$$
C_{s}=C_{0 s}+\beta R^{D-d}
$$

This equation, derived from basic electrical properties of a fractal structure is a more detailed and specific equivalent of Eq. 1, which represents the general principle of fractal analysis approach. This relation clearly shows that for a certain material the $C_{s}$ depends on a structure size and has a power-low dependence on difference of fractal and Euclidean dimension.

In our case the considered fractal structure is the layer adsorbed on the surface of the mercury drop electrode. The size of adsorbed structure is determined by the size of the electrode surface. If the electrode surface is increased, the size (area) of adsorbed structure is increased. Hence, since the size of considered structure ( $R$ in Eq. 19) is described by the area of the mercury drop electrode surface for our case the Eq. (19) becomes: 


$$
C_{s}=C_{0 s}+\beta\left(\frac{A}{\pi}\right)^{(D-2) / 2}
$$

Here, $A=4 \pi r^{2}$, is the electrode surface area, and $r$ is the radius of mercury drop.

\section{Experimental Section}

As a testbed for this theory the behavior of specific capacitance of adsorbed layer of selected organic substances that exhibit fractal structure $^{16,32}$ was examined. The adsorption was studied by electrochemical method and the fractal dimension was determined by using the method of scaling of mercury drop electrode. ${ }^{16}$

To prove the theoretical prediction that the scaling is independent on material (molecular species), or conditions leading to formation of fractal structures we have included measurements on different molecular species and layers formed under different experimental conditions. So, the capacitance of adsorbed fractal layers of two different substances, nonionic surfactant Triton-X-100 (T-X-100) and linoleic acid (LA), was investigated. Capacity current curves were recorded by phase sensitive alternating current voltammetry $\left(90^{\circ}\right.$ out of phase). The $C_{s}$ values were calculate from measured capacity currents obtained for each bulk concentration. The measurements were performed at different experimental conditions: mass-transfer controlled adsorption of T-X-100 in a stirred solution and diffusion controlled adsorption of LA, for 1 and $3 \mathrm{~min}$ accumulation times, respectively. Different experimental conditions were included in this study because it has been found that morphology of fractal adsorbed layer is a result 
of growth mechanism which is influenced by hydrodynamics (stirring or diffusion) and by the structure of the solution (monomers, dimers). ${ }^{33}$

The fractal dimension $(D)$ was determined from the size scaling of the hanging mercury drop electrode. ${ }^{16}$ In this method $D$ is related to the capacitive current, that is proportional to the electrode surface area $\left(A=4 \pi r^{2}\right)$, described by the electrode radius $r$, through relation $i_{c} \sim A \sim r^{D} . D$ is obtained from the slope of the log-log plot of $i_{c}$ vs. $r$. The results for fractal dimension determination were elaborated for electrode potential $0.6 \mathrm{~V}$, representing approximately non-polar electrode. For the purpose of fractal analysis the size of the mercury drop electrode in the voltammetric measurements was varied stepwise over nine sizes corresponding to the electrode surface area in the range of 0.88 to $3.82 \mathrm{~mm}^{2}$. Measurement was performed each time with a freshly prepared new drop of a selected size that did not change during the measurement.

T-X-100 (Rohm and Haas, Italy) and LA (Fluka, Switzerland) were used without further purification. Mercury was purified by double distillation under reduced pressure. $\mathrm{NaHCO}_{3}$ (Merck) was used without prior purification. $\mathrm{NaCl}$ (Kemika, Croatia) was purified by prolonged heating at $450^{\circ} \mathrm{C}$. All solutions were prepared with deionised water obtained with the Milly-Q Water System (Millipore, Switzerland). Carbonate buffer was used to maintain $\mathrm{pH}$ 8.3. The other experimental conditions and the equipment used were the same as in ref. 16. 


\section{Results and discussion}

The changes of the adsorbed layer fractal dimension with increase of T-X-100 and LA bulk concentrations, corresponding to different fractional electrode coverage, and for different adsorption regimes are shown in Figure 1. The observed variations of $D$ depend on the details of the adsorption process.

In the Figure 2 dependence of $C_{s}$ on electrode size is shown for systems with dimension $D=\mathrm{d}=2$, and for a system with fractal dimension $D \approx 2.3$.

We have considered pure electrode surface and surface with adsorbed layer with $D$ $=2$. For such system our theory predicts constant, size independent $C_{s}$. The measurements in the pure electrolyte $(0.55 \mathrm{M} \mathrm{NaCl})$ are included to demonstrate the precision of measurements and agreement with theory of a non-fractal, well-known and controlled system. For the pure electrolyte the measured $D=1.999 \pm 0.005$, reflects a smooth electrode surface. The average measured value of $C_{S}$ is $0.222 \pm 0.001 \mathrm{~F} / \mathrm{m}^{2}$ complying with typical experimental values of such systems. ${ }^{34}$ The slight deviation of the measured points from the straight line represent the scatter of the measurement data due to manual adjustment of mercury drop size.

The curve 2 represents $C_{s}$ obtained for adsorbed layers of T-X-100 with bulk concentration of $4.0 \mathrm{mg} / \mathrm{dm}^{3}$, with $D \approx 2$. As we can see the obtained $C_{s}$ value is constant and independent on size. This is in perfect agreement with our theoretical prediction for structures with $D=\mathrm{d}=2$, and also corresponds to the generally accepted concept of size-independent $C_{s}$. The decrease of capacitance of adsorbed layer in respect to pure electrolyte is a consequence of adsorption of organic molecules on the electrode that have lower dielectric constant than the electrolyte. Hence, the increase in 
electrode surface coverage, as described by corresponding isotherm, results in decrease of capacitance in a non-linear fashion.

The curve 3 represents measured data and corresponding fit with Eq. 20 for a system with distinct fractal dimension $D=2.29$ obtained for adsorbed layer of LA at bulk concentration $0.77 \mathrm{mg} / \mathrm{dm}^{3}$. In the fit of measured $C_{S}$ vs. $A$ data, the corresponding experimental $D$ value has been used along with $C_{s 0}$ and $\beta$ as fit parameters in the least square method. In the fit procedure the values of $D$ and $A$ were also allowed to vary slightly within the limits corresponding to the respective standard deviations. The experimental data (depicted by symbols) are well fitted with theoretical curve based on Eq. 20.

Theoretical considerations (Eq. 20) predict that the specific capacitance $\left(C_{S}\right)$ of the fractal layer is not constant but changes with structure size (in our case with electrode size i.e. adsorbed layer area) in a way determined by a fractal dimension regardless of other parameters governing the adsorption. Hence, it is confirmed that for adsorbed layers with $D \neq 2$, the measured values of specific capacitance show size- and $D$ dependence as predicted by our theory.

The applied experimental electrochemical method is limited to only one order of magnitude in size range but a lot of other systems that are of interest are on a larger or a much smaller scale of size ranges (e.g. in biophysics, nanomaterials etc.). Therefore, to predict the behavior of a system in a much broader size range than measured, one has to extrapolate from the measurement data. In the Figure 3 the behavior of the $C_{s}$ vs. $A$ in measured and extrapolated size ranges is depicted (here $A$ denotes representative size of any considered structure). The boxed area represents experimentally investigated size range. Here, the $C_{s}$ of different T-X-100 layers are shown. Curves 1 and 2 represent fit of measured data with Eq. 20, for two adsorbed layers that have same $D \neq 2$. However, 
these layers are obtained at the different adsorption stages of the layer formation, corresponding to the T-X-100 bulk concentration 0.09 and $0.5 \mathrm{mg} / \mathrm{dm}^{3}$, respectively (cf. Figure 1). Although the $D$ and size range are the same, it can be seen that the curvature of curve 1 is greater than curve 2. This is a consequence of difference in factors $\beta$ $(\beta=\alpha k / l)$ entering into the expression for the curvature and corresponding to different average layer thickness. The curve 3 corresponds to the fit for the layer with the highest observed $D$, obtained at the bulk concentration $0.26 \mathrm{mg} / \mathrm{dm}^{3} \mathrm{~T}-\mathrm{X}-100$. It can be seen that for the investigated size range the rate of change of $C_{s}$ (curve inclination) is greater for higher $D$ as predicted.

The dynamics of change of $C_{s}$ with changes in $D$ is different and depends on a considered size range. As the structure size range decreases the rate of $C_{s}$ change increases. With the increase of the fractal structure size the changes are more pronounced for the systems with higher fractal dimension.

It is worth noting that the fit of experimental data with Eq. 20 facilitates the evaluation of the average layer thickness, $l$, from the $C$-axis intercept. For example, for a T-X-100 layer obtained at bulk concentration of $0.26 \mathrm{mg} / \mathrm{dm}^{3}$ with $D=2.52$ (curve 3 in Figure 3) the fit to the measured data gave intercept value of $0.00529 \mu \mathrm{F} / \mathrm{mm}^{2}$, wherefrom $l=1.67 \mathrm{~nm}$, what is compatible with $\mathrm{T}-\mathrm{X}-100$ molecule size. This fit is very sensitive in regard to the experimental data scatter that results from experimental and measurement errors. Hence, to exactly calculate $l$ the measurements must be done very precisely, especially in regard to mercury drop size determination and if possible should be extended to more than one order of magnitude. 


\section{Summary and Conclusions}

The behavior of relative permittivity and consequently specific capacitance of fractal structures in regard to its size and fractal dimension is considered. The theory of relative permittivity of fractal structures is developed. It predicts that relative permittivity (dielectric constant, $\kappa$ ) is not constant but rather depends on size and fractal dimension of considered structure. As a consequence of this dependence specific capacitance, $C_{S}$, of fractal structures is not constant for a given material, but exhibits dependence on fractal dimension and size of the considered structure. This theory has been experimentally verified for fractal structures of adsorbed layers of different organic substances (linoleic acid and nonionic surfactant Triton-X-100) applying electrochemical measurements. Complying with the theoretical prediction for a nonfractal and fractal systems with $D=\mathrm{d}=2$, the measured $C_{s}$ values, obtained for the pure electrolyte (non-fractal system) and for the adsorbed layers with fractal dimension $D=$ 2, proved to be size-and dimension-independent. For fractal systems with $D \neq 2.0, C_{s}$ is $D$ - and size-dependent as predicted. The rate of change of $C_{S}$ depends on magnitude of the fractal dimension $D$, size of the fractal structure and factor $\beta$ comprising the material and geometrical features.

Acknowledgement. The authors acknowledge support from the Croatian Ministry of Science and Technology, grants No. 0098029 and No. 0098122. 


\section{References}

(1) Weaver, J. C.; R. D. Astumian, Science 1990, 247, 459.

(2) Procopio, J.; Fornés, J. A., Phys. Rev. E. 1995, 51, 829.

(3) Fornés, J. A., J. Colloid Interface Sci. 1997, 186, 90.

(4) JProcopio, J.; Fornés, J. A., Phys. Rev. E. 1997, 55, 6285.

(5) Fornés, J. A., Phys. Rev. E. 1998, 57, 2110.

(6) Fornés, J. A., Phys. Rev. E. 1998, 57, 2104.

(7) Fornés, J. A., J. Colloid Interface Sci. 2000, 222, 97.

(8) Fornés, J. A.; Ito, A. S.; Curi, R.; Procopio, J. J. Phys. Chem. 1999, 122, 5133.

(9) Hille, B. Ionic Channels of Excitable membranes, Sinauer Associates Inc. Publishers, Sunderland, MA 1992.

(10) DeFelice, L.J. Introduction to Membrane Noise, Plenum press, New York, 1981.

(11) Lauger, P., Physiol. Rev. 1987, 67, 1296.

(12) Zwierzykowski, W.; Knopacka-Lyskawa, D., J. Colloid Interface Sci. 1999, $218,265$.

(13) Dynarowicz P.; Paluch, M., J. Colloid Interface Sci. 1989, 129, 379.

(14) Schuhmann, D., J. Colloid Interface Sci. 1990, 134, 152.

(15) Mandelbrot, B. B. The Fractal Geometry of Nature, Freeman: San Francisco, 1982.

(16) Risović, D.; Gašparović, B.; Ćosović, B., Langmuir 2001, 17, 1088.

(17) Möbius, D.; Dhathathreyan, A.; Kozarac, Z.; Loschek, R.; Müller, A., Adsorption of dyes and proteins to monolayers. Biosensors Applications in Medicine, in Environmental Protection and Process Control (R.D. Schmid and F. Scheller, eds.), GBF Monographs, 1989, Volume 13, pp. 331-338.

(18) Dekany, G.; Csoka, I.; Eros I., J. Dispersion Sci. Technol. 2001, 22, 461.

(19) van Duijvenbode, R. C.; Koper, G. J. M., J. Phys. Chem. B. 2001, 105, 11729.

(20) Chatellier, X.; Bottero, J. Y.; Le Petit, J., Langmuir 2001, 17, 2791.

(21) Subramanian, R.; Lakshminarayanan, V., Corrosion Sci. 2002, 44, 535.

(22) Conway, B. E.; Ayranci, E.; Al-Maznai, H., Electrochim. Acta 2001, 47, 705.

(23) Manne, S.; Gaub, H. E., Science 1995, 270, 1480.

(24) Teschke, O.;Ceotto, G.; de Souza, E. F., Chem. Phys. Lett. 2001, 344, 429. 
(25) Pajkossy, T., J. Electroanal. Chem. 1991, 300,1.

(26) Pfeifer, P., New. J. Chem. 1990, 14, 221.

(27) Avnir, D.; Farin, D.; Pfeifer, P., New. J. Chem. 1992, 16, 439.

(28) Cole, M. W.; Holter, N. S., Phys. Rev. B 1986, 33, 8806.

(29) Wandlowski, T.; Pospíšil, L.; J. Electroanal. Chem. 1989, 270, 319.

(30) Pospišil, L., J. Phys. Chem. 1988, 92, 2501.

(31) Javid, M.;.Brown P. M Field Analysis and Electromagnetics, McGraw Hill, New York, 1963, p. 270.

(32) Gašparović, B.; Risović, D.; Ćosović, B. "Fractality of the adsorbed layer of Triton-X-100 on the mercury electrode and its influence on the oxido-reduction processes of o- and p-nitrophenols". 2001 Joint Meeting 200 ${ }^{\text {th }}$ Meeting of the Electrochemical Society $52^{\text {nd }}$ Meeting of International Society of Electrochemistry. 2001, San Francisco, USA.

(33) Risović, D.; Gašparović, B.; Ćosović, B., "Hydrodynamic influence on the fractal morphology of the linoleic acid adsorbed layer at the mercury/electrolyte interface", submitted.

(34) Hamann, C. H.; Hamnett, A.; W. Vielstich: Electrochemistry, Wiley, Weinheim, 1998, p. 111. 


\section{Figure captions}

Figure 1. (a) Dependence of adsorbed layer fractal dimension on bulk concentration: curve 1 - T-X-100, 1 min stirring ( $\Delta$ ), and curve 2 - LA, 3 min diffusion (o); (b) The corresponding total fractional electrode coverage for T-X-100 (curve 1) and LA (curve 2) vs. respective bulk concentration. Results are elaborated for the electrode potential E $=-0.6 \mathrm{~V}$. Symbols denote measurement points while lines represent the fit with the Bspline.

Figure 2. Dependence of $\mathrm{C}_{\mathrm{s}}$ on the mercury drop area for the systems with $D \approx 2.0$ (curves 1 and 2) and for the adsorbed layer with fractal dimensions $D \neq 2$ (curve 3 ). Curve 1-( $\square$ ) represents pure electrolyte and curve 2-( $\Delta$ ) adsorbed layer of T-X-100, at bulk concentration $4.0 \mathrm{mg} / \mathrm{dm}^{3}$. The average $C_{\text {s }}$ values are $0.222 \pm 0.001 \mu \mathrm{F} / \mathrm{mm}^{2}$, and $0.057 \pm 0.0005 \mu \mathrm{F} / \mathrm{mm}^{2}$, for electrolyte and $\mathrm{T}-\mathrm{X}-100$, respectively. Curve 3-(o) corresponds to linoleic acid, $D=2.29$, at bulk concentration $0.77 \mathrm{mg} / \mathrm{dm}^{3}$. Symbols denote measured values while lines represent corressponding theoretical values calculated from the Eq. 20.

Figure 3. Dependence of $\mathrm{C}_{\mathrm{s}}$ on the structure size and fractal dimension, extrapolated for the extended size range from the measured data of T-X-100 (symbols) fitted with Eq. 20 (lines). Depicted are curves corresponding to $D=2.11$, at bulk concentration 0.09 $\mathrm{mg} / \mathrm{dm}^{3}$ (curve 1), $D=2.11$, at $0.5 \mathrm{mg} / \mathrm{dm}^{3}$ (curve 2), $D=2.52$, at $0.26 \mathrm{mg} / \mathrm{dm}^{3}$ (curve 3). The boxed area represents experimentally investigated size range. Inserted is enlerged small size range comprising $A=0-0.3 \mathrm{~mm}^{2}$. 
Figure 1.

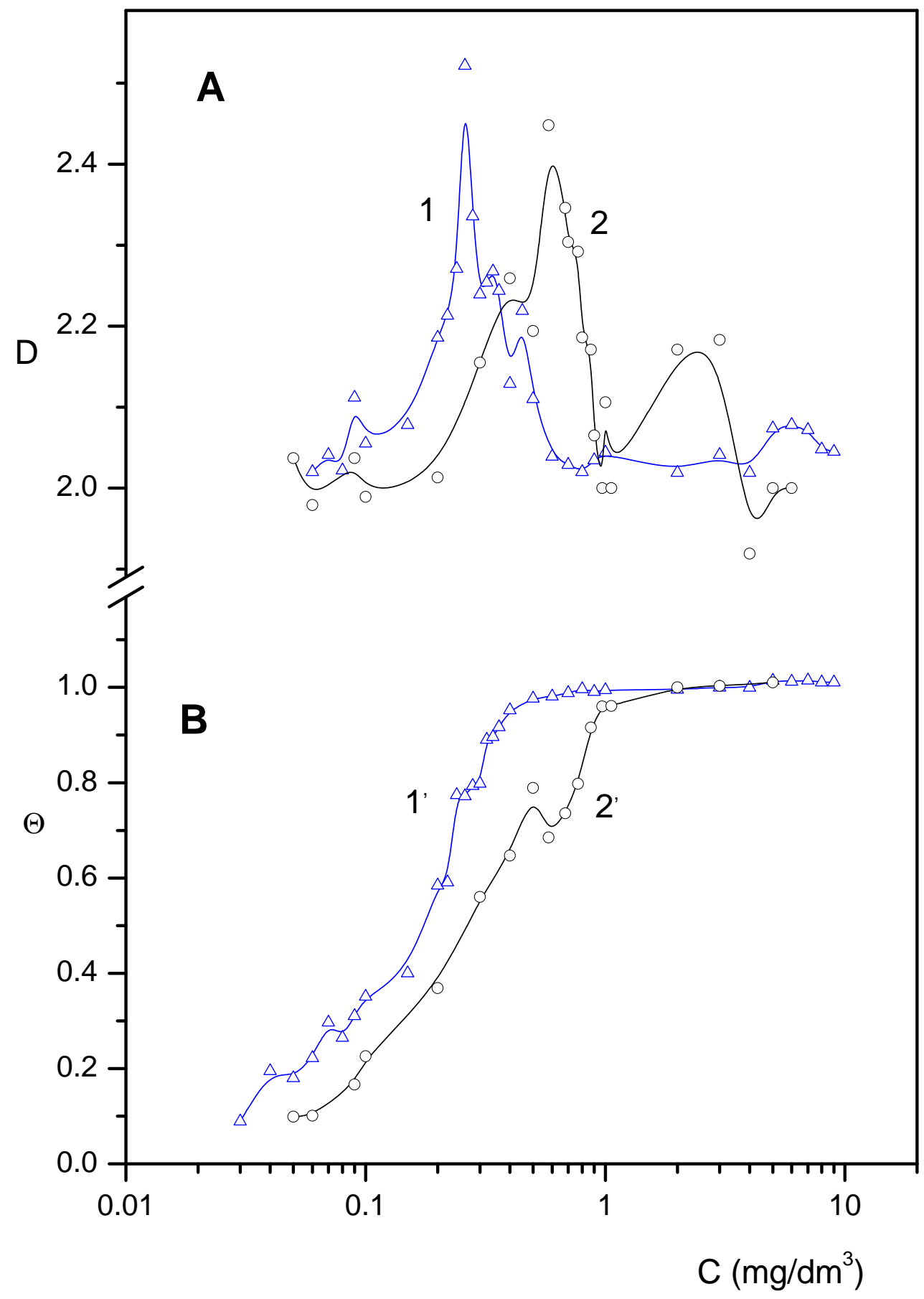


Figure 2.

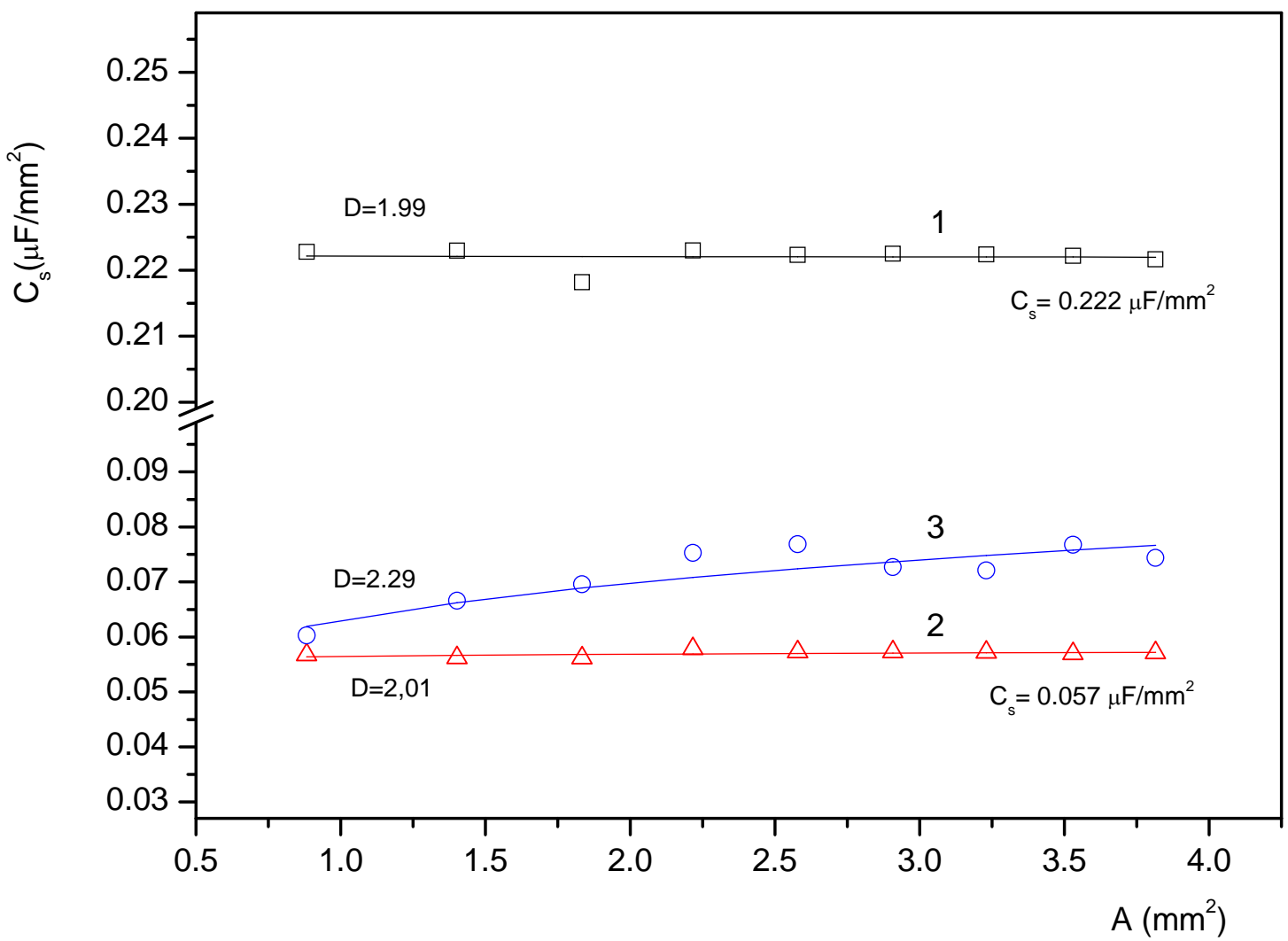


Figure 3.

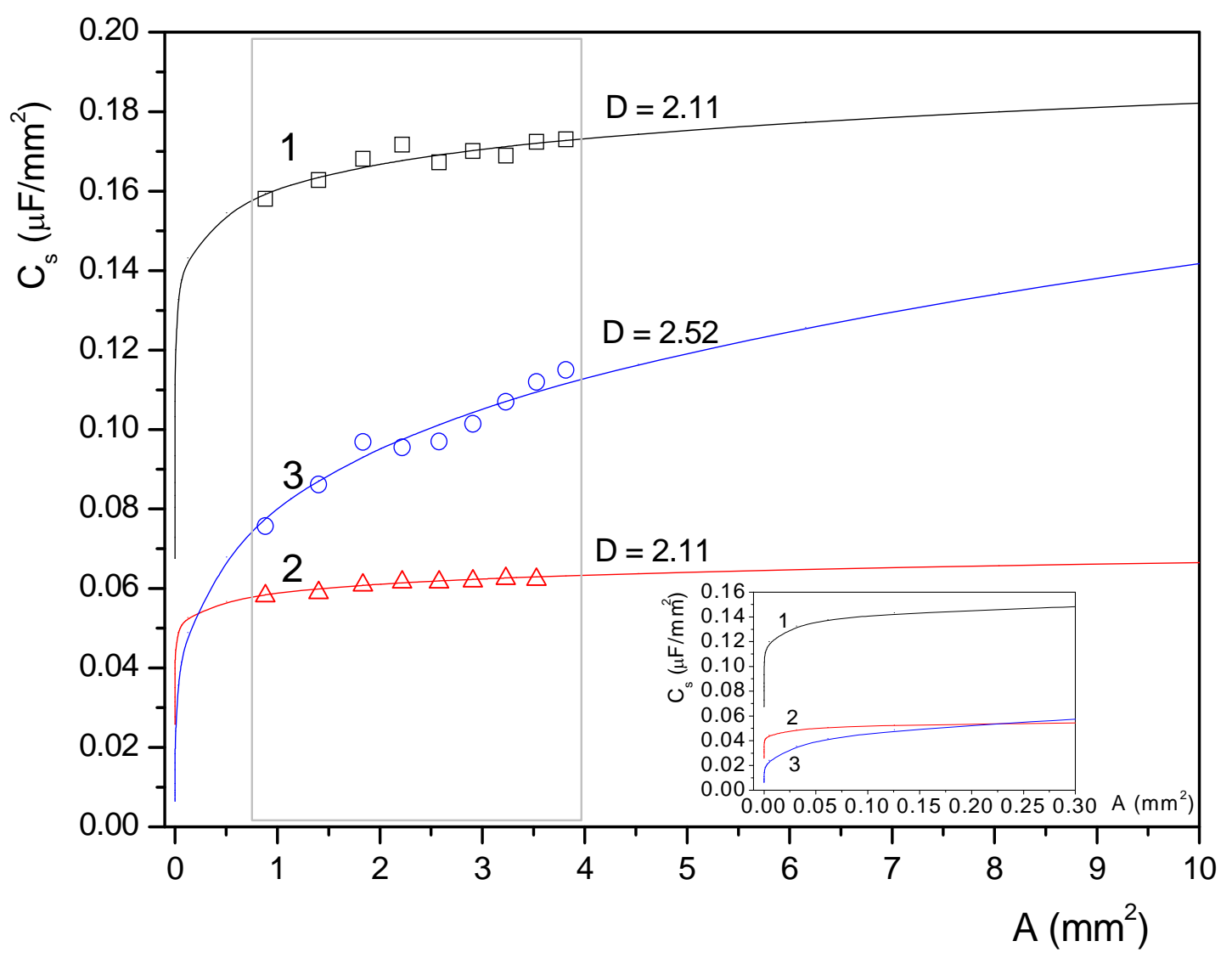

